\title{
Evaluation of the objective Posturo-Locomotor-Manual method in patients with Parkinsonian syndromes
}

\author{
Theresa Zackrisson $^{1}{ }^{*}$, Filip Bergquist ${ }^{2,3}$, Björn Holmberg ${ }^{1,3}$, Bo Johnels ${ }^{1}$ and Thorleif Thorlin ${ }^{1,4}$ \\ 1 Department of Clinical Neuroscience and Rehabilitation, Institute of Neuroscience and Physiology, University of Gothenburg, Gothenburg, Sweden \\ 2 Department of Pharmacology, Institute of Neuroscience and Physiology, University of Gothenburg, Gothenburg, Sweden \\ ${ }^{3}$ Department of Neurology, Sahlgrenska University Hospital, Gothenburg, Sweden \\ ${ }^{4}$ Department of Neurological Rehabilitation, Sahlgrenska University Hospital, Gothenburg, Sweden
}

Edited by:

Giovanni Albani, Istituto Auxologico Italiano, IRCCS, Italy

\section{Reviewed by:}

Alfonso Fasano, Catholic University, Italy

Cristoforo Comi, University of Eastern Piedmont, Italy

\section{${ }^{*}$ Correspondence:}

Theresa Zackrisson, Department of

Clinical Neuroscience and

Rehabilitation, Institute of

Neuroscience and Physiology,

University of Gothenburg, Per

Dubbsgatan 14, 41345 Gothenburg,

Sweden

e-mail: theresa.zackrisson@

neuro.gu.se
Objective methods for quantifying patients' movement capacity would be useful in evaluating progression and interventions in neurodegenerative diseases. The Posturo-LocomotorManual (PLM) test is a standardized automated movement test developed to measure hypokinetic movements in patients with Parkinsonism. Our hypotheses were that the PLM movement time (MT) correlates with the Unified Parkinson's disease rating scale (UPDRS III) motor section, and that the components of the PLM test correlate with the corresponding constructed domains of UPDRS III. We also evaluated the coherence between the results of the two assessment methods after a test dose of levodopa (L-DOPA). We assessed motor function using the PLM method and UPDRS III in parallel, in the absence of medication and after administration of $200 \mathrm{mg}$ L-DOPA, in 73 patients with moderate to advanced Parkinsonism: 47 with Parkinson's disease (PD), 17 with multiple system atrophy (MSA), and 9 with progressive supranuclear palsy (PSP). There was a fair correlation between the two assessment tools in the PD patients but not in the MSA or PSP patients. In the full dataset, there was a fair to good correlation between UPDRS III and the PLM MT. At group level, the UPDRS III L-DOPA test differentiated PD from MSA/PSP, whereas the PLM L-DOPA test differentiated between all three diagnoses.

Keywords: Parkinson's disease, Parkinsonism, movement disorders, L-DOPA test, optoelectronic movement analysis, PLM test, UPDRS III

\section{INTRODUCTION}

The clinical diagnosis of Parkinson's disease (PD) requires bradykinesia and at least one of resting tremor, rigidity, or loss of postural reflexes $(1,2)$. A number of different scales have been used to grade the extent of motor and non-motor symptoms and progression of $\mathrm{PD}(3)$. The unified Parkinson's disease rating scale (UPDRS) is currently the most widely used and evaluated scale, and is the gold standard in clinical PD research $(4,5)$. It is used internationally as a clinical tool to asses Parkinsonism (6), as a marker for disease progression (7), and to evaluate the outcome of interventions (8).

As a complement to a clinical examination, objective methods have been proposed, such as gait quantification and timed tests (e.g., pronation-supination test, finger dexterity, and stand-walksit test) $(9,10)$. Appropriate symptom evaluation methods are essential, both for monitoring disease progress and for evaluating the efficacy of a growing number of treatment interventions. For motor symptoms, rater-independent quantitative evaluation methods that allow repeated measurements are desirable (11). One advantage of using objective quantitative assessment is that it reduces the elements of bias or error that can enter into an assessment process (12), such as inter-rater variability (depending upon the personal skills and experience of each examiner) and intra-rater variability (13).

Another important factor is the timing of symptom assessments after oral drug administration. The time of medication effect onset depends on the gastric emptying frequency, which may decrease with the progress of PD (14-18). In addition, some patients experience a short-lived worsening of Parkinsonian symptoms for up to $20 \mathrm{~min}$ after L-DOPA uptake (19). Assessing symptoms at the right time point is therefore not a trivial task. Timing is less crucial when using continuous or repeated symptom evaluation methods, as these methods record the optimal effect as well as the effect duration. Another aspect of repeated measurements is that they provide information about symptom variability, which makes it possible to decide if a medication-induced change in performance is also statistically significant in individual patients.

The Posturo-Locomotor-Manual (PLM) method was designed to quantify, in a single test, the movement aspects of postural control, locomotion, and goal-directed hand movements and the efficacy with which these movements integrate to a smooth dynamic performance $(20,21)$. This test is automated and raterindependent, and although it tests a limited set of movements, the movements are chosen to reflect an everyday situation and to sample capacities in several domains including balance, locomotion, and basic manual function. The PLM test has been used for more than 10 years in some Swedish neurological clinics to consecutively evaluate hypokinetic symptoms in PD patients and L-DOPA responsiveness, and it is now a commercial product. However, it has not been validated against other methods, and in particular not against UPDRS III, which is the current gold standard for assessing motor symptoms in $\operatorname{PD}(5,22,23)$. 
We hypothesized that the PLM method correlates with the UPDRS III, and that the different phases (Postural, Locomotor, and Manual) of the PLM test correlate with constructed domains of the UPDRS III. We also hypothesized that a significant improvement in the PLM test after a test dose of L-DOPA predicts an improvement in UPDRS III scores.

\section{MATERIALS AND METHODS \\ PATIENTS}

We retrospectively studied clinical rating and PLM measurements in 73 patients with Parkinsonism who were referred to the movement laboratory to perform an L-DOPA test: 47 with PD, 17 with multiple system atrophy (MSA), and 9 with progressive supranuclear palsy (PSP). Patients' characteristics are presented in Table 1.

Motor function was evaluated by the PLM method and UPDRS III in OFF and ON states to determine if the patient responded positively to a test dose of L-DOPA. The patients had given informed consent to the testing procedure before the assessments, and retrospective analysis of the anonymized collected data was approved by the Regional Ethical Review Board in Gothenburg, Sweden.

The PLM results for three PD patients with freezing of gait phenomena that yielded very long movement times (MTs) in OFF were omitted from the correlation analysis, as they were clear outliers in the PLM test and might have skewed this analysis. For seven patients, only the total UPDRS III scores were documented in the medical records; the subscores for the different items were not available. Those seven individuals were all PD patients, and were excluded from the correlation analyses between PLM and the different UPDRS III domains (data presented in Table 3).

\section{THE PLM METHOD}

The PLM method is designed to assess movement patterns in patients with hypokinetic syndromes. The test movement is a compound movement involving a postural phase P (rising up), a locomotion phase L (walking), and a manual phase $\mathrm{M}$ (pendulous arm movement and positioning of a test object on a pedestal, Figure 1). An infrared camera system is used to register body movements by tracking the position of six reflective ball markers of $4 \mathrm{~cm}$ diameter. The markers are attached to the patient's head; to the shoulder, arm, hip, and calf of the most severely disabled side of the body; and to the contralateral foot. A seventh marker is located on a test object consisting of a $500 \mathrm{~g}$ metal handle on a cylindrical horizontal plate (24-26). An automated tracking algorithm is used to identify the markers and analyze the recorded data. The total MT is calculated along with the duration of the different movement phases P, L, and M (27), and the software produces a report after a full test session.

At the start of the test, the patient is asked to stand erect with feet together at a clearly marked start position with the test object on the floor beside them. When instructed, the participant lifts the object from the floor, walks forward as quickly as possible, and places the object on a stand located $1.5 \mathrm{~m}$ away at chin height. This movement is performed three times, after which the patient rests for a short while. Three consecutive movements constitute a measurement group. Ten measurement groups are collected to allow the patient to reach a performance plateau. Mean MT, P, L, and $\mathrm{M}$ durations are automatically calculated from the three best consecutive groups. All nine individual measurements from these three groups are used to calculate standard deviations for each variable.

\section{TEST PROCEDURE}

The PLM test and the UPDRS rating were carried out in the same clinical movement laboratory. All antiparkinsonian medication was withheld for at least $12 \mathrm{~h}$ prior to performing the L-DOPA test, as recommended in published guidelines (10). A trained movement disorder physiotherapist administered the motor part of the UPDRS as described by Goetz et al. (6) before the PLM test started (UPDRS III OFF), and $69 \mathrm{~min}( \pm 32 \mathrm{~min})$ after administration of $200 \mathrm{mg}$ of L-DOPA (UPDRS III ON).

A trained biomedical analyst instructed all patients and performed the PLM test. First, 10 baseline groups of PLM measurements were performed, and the three fastest consecutive groups were designated best mean "OFF" performance. Next, the patients were given $200 \mathrm{mg}$ of L-DOPA (Madopar ${ }^{\circledR}, 200 \mathrm{mg}$ ) dispersed in water (28) and allowed to rest for $31 \mathrm{~min}( \pm 20 \mathrm{~min})$. Following this, two consecutive groups of PLM measurements were collected every $10 \mathrm{~min}$ for $2 \mathrm{~h}$ (Figure 2 ) to ensure that measurements were obtained at the time of maximum L-DOPA effect (18). The three fastest consecutive groups of measurement after L-DOPA administration were designated best mean "ON" performance; these occurred $63 \pm 25 \mathrm{~min}$ after drug administration.

Table 1 | Patient characteristics.

\begin{tabular}{llll}
\hline Diagnosis & PD $(\boldsymbol{n}=\mathbf{4 7})$ & MSA (n= 17) & PSP (n=9) \\
\hline Age (mean \pm SD, range) & $61.9 \pm 7.2(52-76)$ & $53.9 \pm 9.0(43-68)$ & $64.7 \pm 10.4(44-75)$ \\
Males/females & $29 / 18$ & $12 / 5$ & $7 / 2$ \\
Hoehn and Yahr on (median, range) & $2.5,1-3$ & & \\
UPDRS OFF (mean \pm SEM, range) & $35.7 \pm 1.7,6-59$ & $31.6 \pm 3.1,15-61$ & $32.7 \pm 2.6,17-46$ \\
UPDRS ON (mean \pm SEM, range) & $19.1 \pm 1.7,2-61$ & $29.7 \pm 3.1,13-60$ & $29.8 \pm 7.0,18-44$ \\
MT OFF (mean \pm SEM, range) & $3.5 \pm 0.4,1.6-19.3$ & $3.8 \pm 0.6,1.8-10.6$ & $8.6 \pm 3.9,2.6-38.7$ \\
MT ON (mean \pm SEM, range) & $2.1 \pm 0.1,1.2-4.5$ & $3.6 \pm 0.5,1.7-8.7$ & $7.9 \pm 3.25,1.8-30.8$ \\
Disease duration (mean $\pm S D)$ & $13.1 \pm 5.7$ & $3.4 \pm 2.1$ & $4.0 \pm 3.6$ \\
Treatment (mg LDE, mean \pm SD) & $1258 \pm 605$ & $492 \pm 525$ & $494 \pm 578$
\end{tabular}




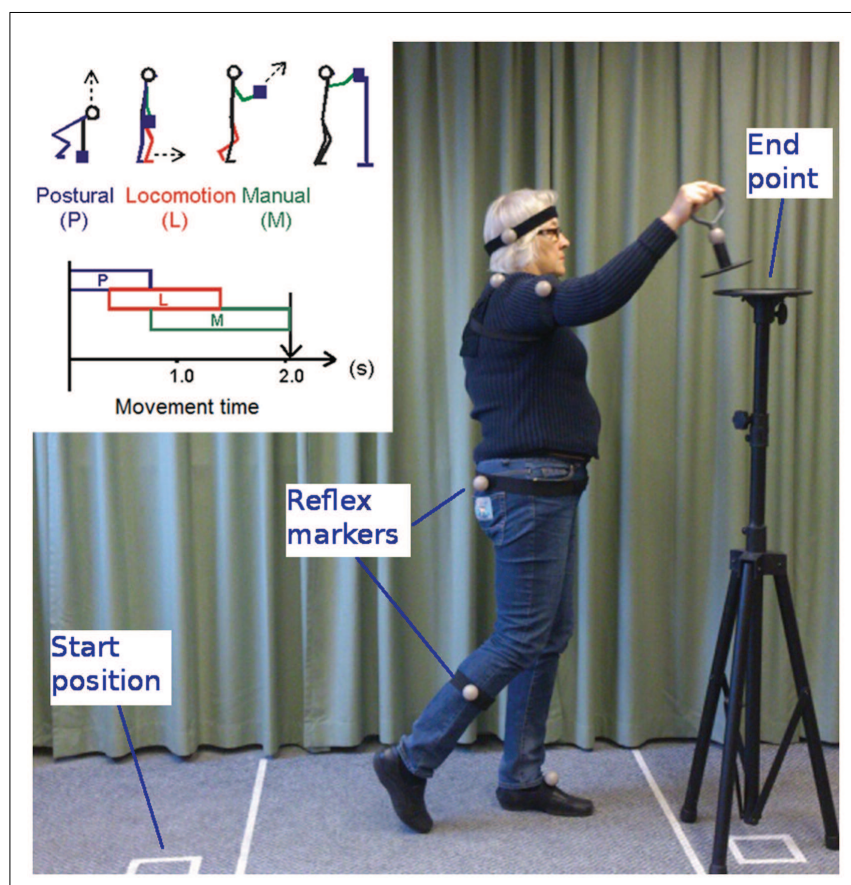

FIGURE 1 | Illustration of the PLM method.

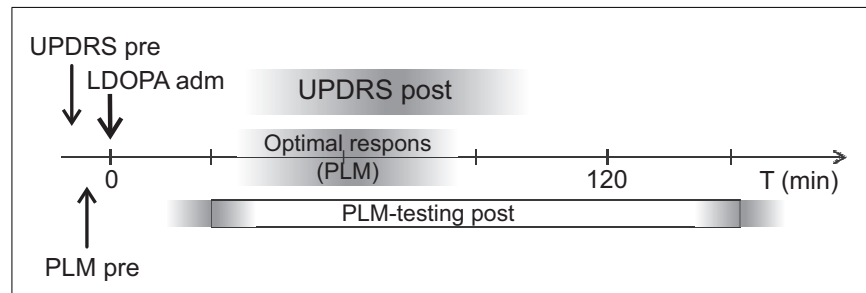

FIGURE 2 |Timeline for the L-DOPA test

\section{UPDRS III DOMAINS AND PLM VARIABLES}

The MT variable of the PLM method was compared to the total score of the UPDRS III (UPDRS items 18-31) as well as to a version of the UPDRS III where scores for speech, facial expression, resting tremor, and postural tremor had been excluded UPDRS $(-)$. We also analyzed the correlations between the different PLM phases and a number of corresponding constructed domains of the UPDRS III (Table 2). The UPDRS III items reflecting postural instability and gait difficulties (PIGD, items 27-30) were evaluated separately against the PLM variables MT, P, and L.

\section{POSITIVE L-DOPA RESPONSE}

Patients who improved by six points or more in UPDRS III score after a test dose of $200 \mathrm{mg}$ L-DOPA were considered to have a positive L-DOPA response (29). We also report the results and concordance between the two tests with some alternative cut off values for acute L-DOPA response: improvements in UPDRS III of 10 points or more, 30 , and 50\%. A change in MT was considered positive if the confidence interval for MT OFF (MT OFF \pm 1.96 SD)
Table 2 | UPDRS III domains and PLM variables.

\begin{tabular}{|c|c|c|}
\hline \multirow[t]{4}{*}{ PIGD* (postural domain + gait) } & Item 27 & Arising from a chair \\
\hline & Item 28 & Posture \\
\hline & Item 29 & Gait \\
\hline & Item 30 & Postural stability \\
\hline \multirow[t]{3}{*}{ Postural domain } & Item 27 & Arising from a chair \\
\hline & Item 28 & Posture \\
\hline & Item 30 & Postural stability \\
\hline Rigidity & Item 22 & Neck \\
\hline \multirow[t]{2}{*}{ Leg domain } & Item 26 & Leg agility \\
\hline & Item 29 & Gait \\
\hline Rigidity & Item 22 & Leg \\
\hline \multirow[t]{3}{*}{ Hand/arm domain ** } & Item 23 & Finger taps \\
\hline & Item 24 & Opening and closing the fist \\
\hline & Item 25 & Pronation and supination \\
\hline Rigidity** & Item 22 & Arm \\
\hline
\end{tabular}

*Postural instability and gait difficulty score.

**Most affected side.

was numerically higher and disjoint from the confidence interval for MT ON (MT ON $\pm 1.96 \mathrm{SD})$.

\section{STATISTICAL METHODS}

Mean, SD, SEM, median, and range of data were used for descriptive purposes. All correlation analysis was performed using Spearman's non-parametric correlation coefficient. All $p$-values were two-tailed and conducted at the 5\% significance level. The following criteria were used to evaluate the strength of the correlations: fair (0.25-0.49), good (0.50-0.74), and excellent ( 0.75 and above) (30). Agreements between UPDRS III and PLM MT L-DOPA tests were tested with McNemar's test after categorizing the response as positive or negative. Analysis of UPDRS III and PLM results at the level of diagnostic groups was done with two-way ANOVA, with diagnosis and treatment state as independent factors and UPDRS III or PLM as the dependent variables. Post hoc comparisons were made with Bonferroni corrected $t$-tests.

\section{RESULTS}

\section{UNIFIED PARKINSON'S DISEASE RATING SCALE III}

The baseline OFF UPDRS III scores and the assessments after a test dose of L-DOPA are given in Table 1 .

The effect of L-DOPA treatment was evaluated by repeated measure two-way ANOVA with diagnosis and treatment state as independent variables and UPDRS III as the dependent variable. There was a significant interaction between diagnosis and treatment state: $F(2,70)=17.5, p<0.0001$, and a significant main effect of treatment state: $F(1,70)=24.2, p<0.001$. The interaction was explained by a reduction in ON UPDRS III scores in PD patients (Figure 3A).

\section{POSTURO-LOCOMOTOR-MANUAL}

The baseline OFF PLM results and the results after a test dose of L-DOPA are given in Table 1 . 

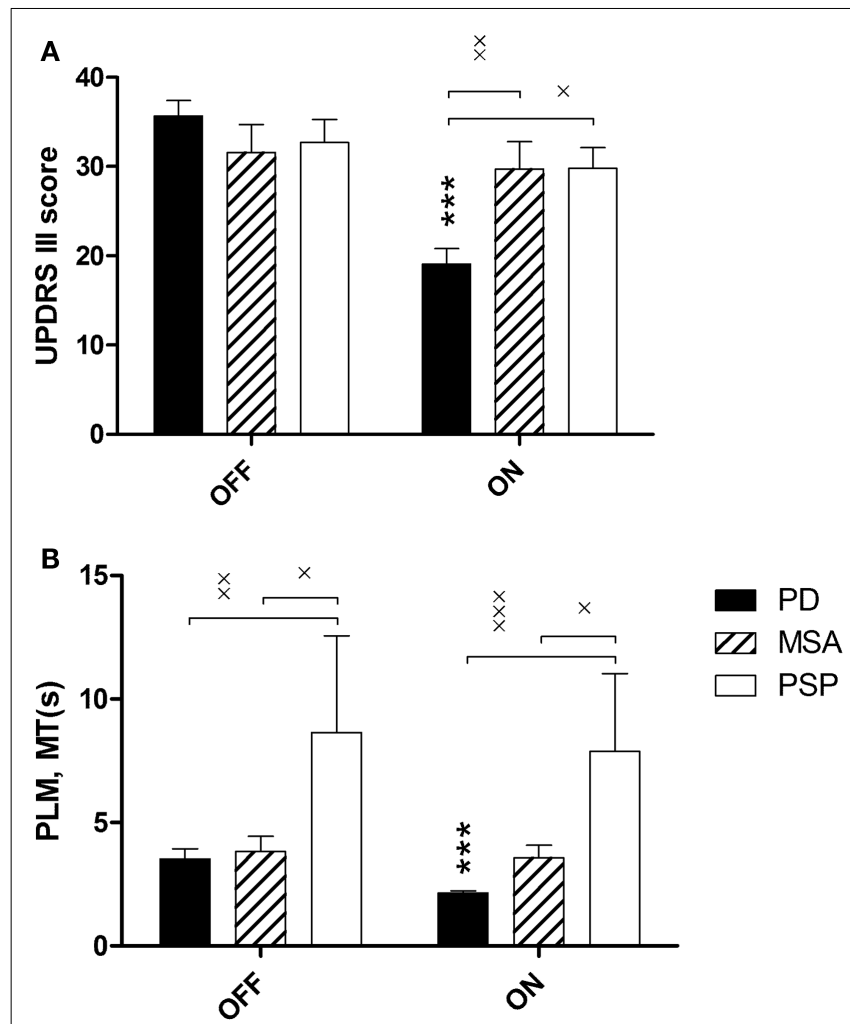

FIGURE 3 | (A) UPDRS III scores before (OFF) and after (ON) $200 \mathrm{mg}$ L-DOPA, stratified over the three diagnoses: Parkinson's disease (PD), multiple system atrophy (MSA), and progressive supranuclear palsy (PSP). (B) PLM mean movement time, MT(s), duration. Main effects of diagnosis and treatment state were analyzed with repeated measure two-way ANOVA followed by Bonferroni corrected $t$-tests where ${ }^{* *} p<0.001, P D$ OFF vs. PD ON, ${ }^{*} p<0.05,{ }^{*}{ }^{*} p<0.01$.

The effect of L-DOPA treatment was evaluated by repeated measure two-way ANOVA using diagnosis and treatment state as independent factors. There was a significant main effect of treatment state: $F(1,70)=5.2, p=0.0258$, and of diagnosis: $F(2,70)=7.1, p=0.0016$. Post hoc analysis revealed that PSP patients had longer MT than the other patient groups in both $\mathrm{ON}$ and OFF, and that MT decreased in PD patients but not in MSA or PSP patients after L-DOPA (Figure 3B).

\section{CORRELATIONS BETWEEN UPDRS III AND THE PLM TEST}

In the full dataset, fair to good correlations were found between most UPDRS III domains and the corresponding PLM phases. There was also significant correlation between the L-DOPA induced effects observed with UPDRS III and PLM (Table 3). Overall, the correlations were lower in OFF than in $\mathrm{ON}$ and OFF-ON (magnitude of change after L-DOPA administration). No or low correlations were found between the $\mathrm{L}$ phase and leg rigidity and between the $\mathrm{M}$ phase and the corresponding constructed UPDRS III domains. Fair and significant correlations between UPDRS III and PLM MT were found with PD patients in OFF, ON, and OFF-ON, but not consistently for MSA and PSP patients, with the exception of OFF-ON in the small sample of PSP patients, where there was excellent correlation (Table 4).

\section{L-DOPA RESPONSE AT SUBJECT LEVEL}

The majority of PD patients responded positively to a test dose of L-DOPA as measured with either method. A decrease of six or more points in UPDRS III classified 40/47 of the PD patients as responders, compared to $34 / 47$ with the PLM method; the concordance between the two test methods was $70 \%$. Few of the MSA patients showed improvement after medication with either method (UPDRS 4/17, PLM 3/17); here, the concordance between the two methods was $59 \%$. In the small sample of PSP patients, about $20 \%$ responded positively to L-DOPA (UPDRS $2 / 9$, PLM 2/9), with a concordance of $78 \%$ between the methods (Table 5).

The Parkinson's disease patients who were discordantly LDOPA negative in the PLM test displayed significantly larger variability in PLM performance before and after L-DOPA administration. The PLM MT standard deviation in this group was $339 \%$ of the L-DOPA induced change in performance, whereas it was $70 \%$ in the group that was L-DOPA responsive with both methods, and $29 \%$ in the group that was positive in the PLM test only [one-way ANOVA of logarithmized values: $F(2,51)=12.5$, $p<0.0001]$. A post hoc $t$-test revealed significant differences between PLM negative/UPDRS positive patients and the other two groups $(p<0.0001$ in both cases $)$.

\section{DISCUSSION}

This study was designed to validate the objective and quantitative PLM method for assessing Parkinsonism, by comparing this method with the commonly used clinical rating scale UPDRS part III. There was a fair correlation between the PLM (MT) and the UPDRS assessment in PD but not in MSA and PSP patients. Overall, the correlations were somewhat stronger in $\mathrm{ON}$ and OFF-ON than in OFF. The postural and locomotive phases of the compound PLM movement showed good correlation to the pre-constructed corresponding domains in the UPDRS when evaluated in the full dataset. However, the movement phase of the PLM test did not correlate with the hand and arm related domains of the UPDRS.

The degree of correlation between the two evaluation methods may appear modest in places, but since our purpose was to examine the relationship between a subjective clinical rating scale and a quantitative analysis of motor function, establishing a fair correlation is a clinically useful validation of the quantitative method. As the PLM method is more expensive than clinical rating, and is also time consuming if the medication response profile is followed beyond maximum effect, a very high correlation would have suggested that the PLM test adds no information beyond that obtained from the UPDRS.

There are several differences between the UPDRS III and the PLM test. In particular, the UPDRS III measures some motor features that go undetected in the PLM test, such as tremor, speech, and facial expression. The PLM test also contains no alternating movements, which may explain the lack of correlation between the arm/hand domain of the UPDRS III and the M phase in the PLM test. We hypothesized that the pendulous arm movement of the $\mathrm{M}$ phase might correspond better to arm rigidity than to 
Table 3 | Correlation between PLM results and the UPDRS in all patients.

\begin{tabular}{|c|c|c|c|c|c|c|c|c|c|}
\hline & \multicolumn{3}{|c|}{ OFF } & \multicolumn{3}{|c|}{ ON } & \multicolumn{3}{|c|}{ OFF-ON } \\
\hline & $r$ & $p$-Value & $n$ & $r$ & $p$-Value & $n$ & $r$ & $p$-Value & $n$ \\
\hline MT vs. UPDRS (total) & 0.37 & 0.0017 & $70^{*}$ & 0.58 & $<0.0001$ & 73 & 0.60 & $<0.0001$ & $70 *$ \\
\hline MT vs. UPDRS (-) & 0.35 & 0.0042 & $64^{* *}$ & 0.56 & $<0.0001$ & $66^{* *}$ & 0.58 & $<0.0001$ & $64 * *$ \\
\hline MT vs. bradykinesia & 0.39 & 0.0013 & 64 & 0.62 & $<0.0001$ & 66 & & & \\
\hline MT vs. PIGD & 0.56 & $<0.0001$ & 64 & 0.62 & $<0.0001$ & 66 & & & \\
\hline P phase vs. postural domain & 0.36 & 0.0030 & 64 & 0.65 & $<0.0001$ & 66 & & & \\
\hline$P$ phase vs. neck rigidity & 0.22 & 0.0806 & 64 & 0.41 & 0.0006 & 66 & & & \\
\hline P phase vs. PIGD & 0.48 & $<0.0001$ & 64 & 0.70 & $<0.0001$ & 66 & & & \\
\hline L phase vs. leg domain & 0.51 & $<0.0001$ & 64 & 0.64 & $<0.0001$ & 66 & & & \\
\hline L phase vs. leg rigidity & -0.16 & 0.2186 & 64 & 0.04 & 0.7474 & 66 & & & \\
\hline L phase vs. PIGD & 0.55 & $<0.0001$ & 64 & 0.53 & $<0.0001$ & 66 & & & \\
\hline M phase vs. hand/arm domain*** & 0.09 & 0.4925 & 64 & 0.29 & 0.0172 & 66 & & & \\
\hline M phase vs. arm rigidity*** & -0.07 & 0.5828 & 64 & 0.12 & 0.3310 & 66 & & & \\
\hline
\end{tabular}

UPDRS (-) excluding scores for speech, facial expression, resting tremor, and postural tremor.

PIGD postural instability gait difficulty score (UPDRS Items 27-30).

*The PLM results for three PD patients in OFF were omitted from the correlation analysis due to freezing of gait phenomena yielding very long movement times.

**UPDRS III subscores were not available for all patients.

***Most affected side.

Table 4 | Correlation between PLM MT(s) and UPDRS III for each diagnosis.

\begin{tabular}{|c|c|c|c|c|c|c|c|c|c|}
\hline & \multicolumn{3}{|c|}{ OFF } & \multicolumn{3}{|c|}{ ON } & \multicolumn{3}{|c|}{ OFF-ON } \\
\hline & $r$ & $p$-Value & $n$ & $r$ & $p$-Value & $n$ & $r$ & $p$-Value & $n$ \\
\hline PD & 0.47 & 0.0013 & $44^{*}$ & 0.44 & 0.0019 & 47 & 0.47 & 0.0015 & $44^{*}$ \\
\hline MSA & 0.49 & 0.0448 & 17 & 0.46 & 0.0635 & 17 & 0.05 & 0.8544 & 17 \\
\hline PSP & 0.27 & 0.4860 & 9 & 0.22 & 0.5755 & 9 & 0.75 & 0.0210 & 9 \\
\hline
\end{tabular}

*The PLM results for three PD patients in OFF were omitted from the correlation analysis due to freezing of gait phenomena yielding very long movement times.

hand bradykinesia, but this assumption was not supported by the correlation analysis.

The presence of tremor strongly influences the UPDRS III scores, but has no practical effect in PLM. Nevertheless, removing tremor and facial features from UPDRS III did not improve the correlation between UPDRS III and PLM MT. This suggests that the tremor rating does not contribute fundamentally different information from that captured by PLM. Consequently, it appears that the PLM test gives a fair to good estimate of overall Parkinson symptomatology, but it is clear that the PLM test covers fewer domains of Parkinsonism and its face validity is therefore lower than that of UPDRS (7). The advantage of the PLM test is the rater-independent outcome and the repeated measurements that eliminate inter-rater variability and provide a measure of the patient's performance and symptom variability. As a consequence, it is possible to determine whether a treatment has a significant effect in a single patient. It is also our experience that, in some cases, being able to present objective improvements over time has a pedagogical value and may improve compliance.
We registered significant improvements in PD patients following acute L-DOPA treatment with both the UPDRS III and the PLM method, but no such improvements were seen in the MSA or PSP group. Interestingly, the PLM test and the UPDRS III provided different information on a group level, as PSP patients had significantly higher MT than the other diagnostic groups but were not singled out by their UPDRS III scores. However, the PSP group was small, and the significantly higher MT needs to be confirmed in a larger population with atypical Parkinsonism.

Different cut off values for UPDRS improvements have been used to categorize subjects as L-DOPA responsive or not. Schrag et al. (29) suggested that a decrease of more than five points in UPDRS III after L-DOPA administration represents a minimal clinically relevant improvement in motor ability. Others have defined responders as those improving by $30 \%$ in UPDRS III score (31); however, the outcome then largely depends on baseline score, so more advanced patients have lesser probability of demonstrating a positive effect. Because a clinically relevant improvement is the most appropriate reason for introducing or continuing treatment, we argue that a six-point cut off is preferable. This is further corroborated by our finding that the 6-point cut off identified more L-DOPA responders than a 10 -point, 30 , or $50 \%$ improvement in UPDRS III, and had the best overlap with the PLM method (Table 5).

Because the PLM test covers a smaller subset of L-DOPA responsive features, the somewhat lower ratio of L-DOPA responders revealed by the PLM method in our material was as expected. However, it was also evident that the group of patients who were L-DOPA-responsive with UPDRS III, but not PLM, displayed significantly larger variability in PLM MT both in OFF and ON, whereas patients who were positive only with the PLM test had a significantly lower variability in $\mathrm{MT}_{\mathrm{ON}}$. Patients who were congruent in both tests showed low variability both in OFF and ON. 
Table 5 | L-DOPA responses with the two measuring tools.

\begin{tabular}{|c|c|c|c|c|c|c|c|c|c|c|c|c|}
\hline \multirow[t]{2}{*}{ UPDRS improvement cut off } & \multicolumn{4}{|c|}{$\operatorname{PD}(n=47)$} & \multicolumn{4}{|c|}{$\operatorname{MSA}(n=17)$} & \multicolumn{4}{|c|}{ PSP $n(9)$} \\
\hline & $\geq 6 p$ & $\geq 10 p$ & $\geq 30 \%$ & $\geq 50 \%$ & $\geq 6 p$ & $\geq 10 p$ & $\geq 30 \%$ & $\geq 50 \%$ & $\geq 6 p$ & $\geq 10 p$ & $\geq 30 \%$ & $\geq 50 \%$ \\
\hline Positive in PLM & 34 & 34 & 34 & 34 & 3 & 3 & 3 & 3 & 2 & 2 & 2 & 2 \\
\hline Negative in PLM & 13 & 13 & 13 & 13 & 14 & 14 & 14 & 14 & 7 & 7 & 7 & 7 \\
\hline Positive in UPDRS & 40 & 35 & 34 & 28 & 4 & 0 & 1 & 0 & 2 & 0 & 0 & 0 \\
\hline Negative in UPDRS & 7 & 12 & 13 & 19 & 13 & 17 & 16 & 17 & 7 & 9 & 9 & 9 \\
\hline Concordant positive in PLM (\%) & 64 & 57 & 55 & 47 & 0 & 0 & 0 & 0 & 11 & 0 & 0 & 0 \\
\hline Concordant negative in PLM (\%) & 6 & 11 & 11 & 15 & 59 & 82 & 76 & 82 & 67 & 78 & 78 & 78 \\
\hline Discordant (\%) & 30 & 32 & 34 & 38 & 41 & 18 & 24 & 18 & 22 & 22 & 22 & 22 \\
\hline
\end{tabular}

This was an unexpected but interesting finding, indicating that the PLM method has the ability to detect variability in motor performances both OFF and ON medication, and that the variability differs between patients; some patients have a large variability in OFF, others have a large variability in both OFF and ON, and yet others have a very small variability after administration of LDOPA as compared to before administration of L-DOPA. This may to some extent explain why the PLM test is less sensitive than UPDRS III in detecting L-DOPA improvement, because for a positive L-DOPA response in the PLM test the improvement has to be statistically significant, but with UPDRS it only has to be more than five points.

This raises the question of the best timing of the assessment after an L-DOPA dose. The repeated measurements obtained with the PLM method reveal highly variable performances in some patients. The variability in performance will not be detected if a single UPDRS rating is performed before and at a defined time point after treatment $(15,18,32,33)$. This may be an advantage of the PLM test, but the clinical relevance of detecting motor performance variability is to our knowledge not known. The repeated PLM tests indicated that optimal improvement after LDOPA occurred $63 \pm 25 \mathrm{~min}$ after administration, so a 1-h wait after administering L-DOPA dispersed in water before evaluation of effect appears appropriate. All UPDRS III ratings ON medication in the present study were performed within the optimal time span.

One obvious problem with the PLM test is that patients who cannot walk cannot perform the test. Also, patients who experience freezing of gait phenomena may produce disproportionately long MTs. Although this does not preclude assessment of L-DOPA response in patients who can walk after the test dose, it introduces a non-linear component to the PLM test. In the present dataset, we excluded 3 out of 73 patients due to obvious freezing of gait in

\section{REFERENCES}

\footnotetext{
1. Hoehn MM, Yahr MD. Parkinsonism: onset, progression and mortality. Neurology (1967) 17:427-42. doi: 10.1212/WNL.17.5.427

2. Jankovic J. Parkinson's disease: clinical features and diagnosis. $\mathrm{J} \mathrm{Neu}$ rol Neurosurg Psychiatry (2008) 79:368-76. doi:10.1136/jnnp.2007. 131045
}

OFF. Most of these patients could still be assessed in ON, and the exclusion was made only because they were outliers in the correlation analysis. Despite this general limitation, the PLM test may still be useful for evaluating whether freezing of gait is L-DOPA responsive in individual patients.

The current study population had relatively long mean disease duration, and had passed the early stages when correct diagnosis is more difficult. A prospective study of patients who have recently presented with their first signs of hypokinetic movement disorders would be needed to determine whether the PLM test can provide any additional diagnostic information that might aid the clinician.

\section{CONCLUSION}

We found a fair correlation between the PLM test and a simultaneous UPDRS III rating in PD patients OFF and ON medication, despite the fact that the PLM test only samples some of the disabilities rated with the UPDRS III. On a group level, the PLM method distinguished between PD, MSA, and PSP patients. The $\mathrm{PD}$ patients could be identified by a positive response to L-DOPA, and the PSP group was significantly slower than both MSA and PD patients. The PLM method provides a measure of motor performance variability ON and OFF medication that cannot easily be obtained with UPDRS III. Our findings suggest that the PLM test is a valid method for assessing motor performance in OFF and ON state, as well as L-DOPA responsiveness in patients with Parkinsonism at moderately advanced stages. It remains to be seen whether the PLM test can also reliably detect positive L-DOPA responses in de novo PD patients less marked by their disease.

\section{ACKNOWLEDGMENTS}

We thank Barbro Eriksson for her work in performing the PLM tests. This study was funded by the Swedish Federal Government under the LUA/ALF agreement.

NJ: Macmillan Healthcare Information (1987). p. 153-63.

5. Schrag A, Spottke A, Quinn NP, Dodel R. Comparative responsiveness of Parkinson's disease scales to change over time. Mov Disord (2009) 24:813-8. doi:10.1002/mds. 22438

6. Goetz CG, Stebbins GT, Chmura TA, Fahn S, Klawans HL, Marsden CD. Teaching tape for the motor section of the unified Parkinson's disease rating scale. Mov Disord (1995) 10:263-6. doi:10.1002/mds. 870100305

7. Goetz C, Poewe W, Rascol O, Sampaio C, Stebbin G, Fahn S, et al. The unified Parkinson's disease rating scale (UPDRS): status and recommendations. Mov Disord (2003) 18:738-50. doi:10.1002/ mds. 10473 
8. Martinez-Martin P, Gil-Nagel A, Gracia LM, Gomez JB, MartinezSarries J, Bermejo F. Unified Parkinson's disease rating scale characteristics and structure. The Cooperative Multicentric Group. Mov Disord (1994) 9:76-83. doi:10.1002/ mds.870090112

9. Langston JW, Widner H, Goetz CG, Brooks D, Fahn S, Freeman $\mathrm{T}$, et al. Core assessment program for intracerebral transplantations (CAPIT). Mov Disord (1992) 7:213. doi: $10.1002 / \mathrm{mds} .870070103$

10. Defer GL, Widner H, Marie RM, Remy P, Levivier M. Core assessment program for surgical interventional therapies in Parkinson's disease (CAPSIT-PD). Mov Disord (1999) 14:572-84. doi:10.1002/15318257(199907)14:4<572::AIDMDS1005>3.0.CO;2-C

11. Garcia Ruiz PJ, Muñiz de Igneson J, Ayerbe J, Frech F, Sánchez Bernardos V, Lopez Ferro O, et al. Evaluation of timed tests in advanced Parkinsonian patients who were candidates for subthalamic stimulation. Clin Neuropharmacol (2005) 28:157. doi:10.1097/01.wnf.0000150869. 44630.71

12. Turnbull GI. Physical Therapy Management of Parkinson's Disease. New York: Churchill Livingstone (1992).

13. Post B, Merkus MP, de Bie RM, de Haan RJ, Speelman JD. Unified Parkinson's disease rating scale motor examination: are ratings of nurses, residents in neurology, and movement disorders specialists interchangeable? Mov Disord (2005) 20:1577-84. doi:10.1002/ mds. 20640

14. Johnels B, Ingvarsson PE, Holmberg B, Matousek M, Steg G. Single-dose L-dopa response in early Parkinson's disease: measurements with optoelectronic recording technique. Mov Disord (1993) 8:56-62. doi: $10.1002 / \mathrm{mds} .870080111$

15. Colosimo C, Merello M, Hughes AJ, Sieradzan K, Lees AJ. Motor response to acute dopaminergic challenge with apomorphine and levodopa in Parkinson's disease: implications for the pathogenesis of the on-off phenomenon. J Neurol Neurosurg Psychiatry (1996) 60:634-7. doi:10.1136/jnnp. 60.6.634

16. Müller T, Woitalla D, Saft C, Kuhn W. Levodopa in plasma correlates with body weight of Parkinsonian patients. Parkinsonism Relat Disord (2000) 6:171-3. doi:10.1016/S13538020(00)00005-5

17. Haussermann P, Kuhn W, Przuntek $\mathrm{H}$, Muller T. Integrity of the blood-cerebrospinal fluid barrier in early Parkinson's disease. Neurosci Lett (2001) 300:182-4. doi:10.1016/ S0304-3940(01)01574-9

18. Müller T, Benz S, Börnke C, Russ H, Przuntek H. Repeated rating improves value of diagnostic dopaminergic challenge tests in Parkinson's disease. J Neural Transm (2003) 110:603-9. doi:10. 1007/s00702-003-0815-y

19. Merello M, Lees AJ. Beginning-ofdose motor deterioration following the acute administration of levodopa and apomorphine in Parkinson's disease. J Neurol Neurosurg Psychiatry (1992) 55:1024-6. doi: 10.1136/jnnp.55.11.1024

20. Johnels B, Ingvarsson PE, Thorselius M, Valls M, Steg G. Disability profiles and objective quantitative assessment in Parkinson's disease. Acta Neurol Scand (1989) 79:22738. doi:10.1111/j.1600-0404.1989. tb03743.x

21. Steg G, Johnels B. Physiological mechanisms and assessment of motor disorders in Parkinson's disease. Adv Neurol (1993) 60:358-65.

22. Perlmutter JS. Assessment of Parkinson disease manifestations. Curr Protoc Neurosci (2009) 49:10.1.1-10.1.14. doi: 10.1002/0471142301.ns1001s49

23. Racette BA, Tabbal SD, Jennings D, Good LM, Perlmutter JS, Evanoff
BA. A rapid method for mass screening for parkinsonism. Neurotoxicology (2006) 27:357-61. doi: 10.1016/j.neuro.2005.11.005

24. Ingvarsson P, Johnels B, Lund S, Steg G. Coordination of manual, postural, and locomotor movements during simple goal-directed motor tasks in Parkinsonian off and on states. Adv Neurol (1987) 45:37582.

25. Johnels B, Ingvarsson PE, Matousek M, Steg G, Heinonen EH. Optoelectronic movement analysis in Parkinson's disease: effect of selegiline on the disability in de novo Parkinsonian patients - a pilot study. Acta Neurol Scand Suppl (1991) 136:40-3. doi:10.1111/j.1600-0404. 1991.tb05019.x

26. Johnels B, Ingvarsson PE, Steg G, Olsson T. The PosturoLocomotion-Manual test. A simple method for the characterization of neurological movement disturbances. Adv Neurol (2001) 87:91-100.

27. Zackrisson T, Holmberg B Johnels B, Thorlin T. A new automated implementation of the Posturo-Locomotion-Manual (PLM) method for movement analysis in patients with Parkinson's disease. Acta Neurol Scand (2010) 123:274-9. doi: 10.1111/j.1600-0404.2010.01415.x

28. Albanese A, Bonuccelli U, Brefel C, Chaudhuri KR, Colosimo C, Eichhorn T, et al. Consensus statement on the role of acute dopaminergic challenge in Parkinson's disease. Mov Disord (2001) 16:197-201. doi: 10.1002/mds.1069

29. Schrag A, Sampaio C, Counsell N, Poewe W. Minimal clinically important change on the unified Parkinson's disease rating scale. Mov Disord (2006) 21:1200-7. doi:10.1002/ mds. 20914

30. Portney L, Watkins M. Foundations of Clinical Research: Applications to Practice. Englewood Cliffs, NJ: Prentice-Hall (2000).
31. Merello M, Nouzeilles MI, Arce GP, Leiguarda R. Accuracy of acute levodopa challenge for clinical prediction of sustained long-term levodopa response as a major criterion for idiopathic Parkinson's disease diagnosis. Mov Disord (2002) 17:795-8. doi: $10.1002 / \mathrm{mds} .10123$

32. Olanow W, Schapira AH, Rascol O. Continuous dopaminereceptor stimulation in early Parkinson's disease. Trends $\mathrm{Neu}$ rosci (2000) 23:117-26. doi: 10.1016/S1471-1931(00)00030-6

33. Nyholm D, Constantinescu R, Holmberg B, Dizdar N, Askmark H. Comparison of apomorphine and levodopa infusions in four patients with Parkinson's disease with symptom fluctuations. Acta Neurol Scand (2009) 119:345-8. doi: 10.1111/j.1600-0404.2008.01104.x

Conflict of Interest Statement: The research was conducted in the absence of any commercial or financial relationships that could be constructed as a potential conflict of interest.

Received: 19 February 2013; accepted: 07 July 2013; published online: 19 July 2013. Citation: Zackrisson T, Bergquist F, Holmberg B, Johnels $B$ and Thorlin $T$ (2013) Evaluation of the objective Posturo-Locomotor-Manual method in patients with Parkinsonian syndromes. Front. Neurol. 4:95. doi: 10.3389/fneur.2013.00095

This article was submitted to Frontiers in Movement Disorders, a specialty of Frontiers in Neurology.

Copyright (C) 2013 Zackrisson, Bergquist, Holmberg, Johnels and Thorlin. This is an open-access article distributed under the terms of the Creative Commons Attribution License, which permits use, distribution and reproduction in other forums, provided the original authors and source are credited and subject to any copyright notices concerning any third-party graphics etc. 